\title{
Quiste epidermoide en cavidad oral. Reporte de un caso
}

\author{
Epidermoid cyst in oral cavity. A case report
}

Sandra Milena Buitrago-Rojas¹ 1 , Gersón Enrique Cruz-Pérez²

Citación: Buitrago-Rojas SM, Cruz-Pérez GE. Quiste epidermoide en cavidad oral. Reporte de un caso. Ustasalud. 2019;18: 55-59.

Licencia Creative Commons

\section{(c) (1) () $\Theta$}

( alterarlos, adicionalmente se debe reconocer la autoría de las personas que figuran en las publicaciones, pero estas no podrán ser comercializadas.

\section{Resumen}

El quiste epidermoide es una lesión benigna, no odontogénica, que se presenta con una prevalencia promedio de $1,6 \%$ a $7,0 \%$ del total de los quistes. Se deriva del tejido ectodérmico, cubierto por epitelio escamoso estratificado. La localización más frecuente es en el piso de boca, seguido de lengua, mucosa bucal y labio inferior. Generalmente, es asintomático y de crecimiento lento; su diagnóstico oportuno es de gran importancia, pues con el paso del tiempo podría infectarse y causar complicaciones. Se presenta el caso de un paciente de sexo masculino, 52 años, quien asiste a consulta por sensación de abultamiento en la mucosa bucal inferior izquierda. Se realiza biopsia excisional y análisis microscópico. En los controles posoperatorios no hay evidencia de recurrencia.

Palabras clave: Boca, patología bucal, mucosa bucal.

\section{Abstract}

The epidermoid cyst is a benign, non-odontogenic lesion, with an average prevalence of $1.6 \%$ to $7.0 \%$ of all cysts. It is derived from ectodermal tissue, covered by stratified squamous epithelium. The most frequent location is on the floor of the mouth, followed by the tongue, buccal mucosa and lower lip. It is generally asymptomatic and slow growing. Its timely diagnosis is of great importance, because over time it could become infected causing complications. We present the case of a 52-year-old male patient, who attended a consultation due to the sensation of bulging in the left lower oral mucosa. Excisional biopsy and microscopic analysis are performed. In the postoperative controls there is no evidence of recurrence.

Keywords: Mouth, oral pathology, mouth mucosa.
1 Magíster en Odontología, do-
cente Facultad de Odontología,
Universidad Santo Tomás, Buca-
ramanga, Colombia.
${ }^{2}$ Especialista en Patología y Ci-
rugía Bucal, docente Facultad de
Odontología, Universidad Santo
Tomás, Bucaramanga, Colombia.
Autor de correspondencia:
Sandra Milena Buitrago-Rojas
Correo electrónico:
sandra.buitrago@ustabuca.edu.co

Recibido para publicación:

19 de octubre de 2019

Aceptado para publicación:

30 de noviembre de 2019 


\section{INTRODUCCIÓN}

Los quistes se clasifican histológicamente como epidermoides cuando se derivan del tejido ectodérmico y se encuentran recubiertos por epitelio escamoso estratificado, dermoides cuando poseen una cavidad rodeada por epitelio similar y que contiene estructuras como glándulas sebáceas o sudoríparas; y teratomas cuando la cavidad está cubierta por epitelio que contiene algunos derivados del endodermo y mesodermo, como músculo, mucosa intestinal, mucosa respiratoria, hueso o vasos sanguíneos ${ }^{1-6}$.

El Quiste Epidermoide (QE) es una lesión quística, benigna, poco frecuente $(0,01 \%$ de los quistes de cavidad oral y $1,6 \%$ a $6,9 \%$ de cabeza y cuello) ${ }^{1-4}$. Su etiología es desconocida, sin embargo, se ha asociado a remanentes de tejido embrionario ectodérmico durante la fusión del primer y segundo arco branquial durante el desarrollo embrionario ${ }^{5}$.

El QE puede encontrarse en cualquier parte del cuerpo. A nivel bucal, los sitios afectados en orden de prevalencia son: piso de boca, lengua, labios y mucosa bucal ${ }^{3}$. Clínicamente, se evidencia como una inflamación asintomática, de crecimiento lento y fluctuante. Usualmente se presenta en adultos jóvenes y rara vez en niños ${ }^{2-5,7,8}$.

Los diagnósticos diferenciales del $\mathrm{QE}$ son ránula, malformación linfática, quiste dermoide, teratoma, quiste tirogloso e higroma; siendo más complejo en las lesiones de aspecto quístico que pudieran extenderse al cuello, como las ránulas con extensión cervical, para las cuales el análisis histopatológico es decisivo, pues presenta elementos linfáticos en la cápsula del quiste ${ }^{8-11}$.

Respecto al tratamiento, este varía de acuerdo con la presentación clínica, tamaño y localización. Se ha sugerido la excisión quirúrgica completa por vía intraoral o extraoral, evitando la ruptura de la cápsula, ya que el contenido quístico pudiera actuar como irritante en el tejido fibrovascular y generar inflamación durante la etapa posoperatoria ${ }^{8,12}$.

La recidiva de esta lesión es baja, relacionándose con resecciones incompletas. Por lo tanto, cuando el quiste se elimina totalmente, el pronóstico es favorable. El propósito de este artículo es describir el diagnóstico y tratamiento de un quiste epidermoide localizado en la mucosa bucal izquierda ${ }^{11}$.

\section{REPORTE DE CASO}

Paciente de sexo masculino, 52 años, sin enfermedades o alteraciones sistémicas. Asiste a consulta odontológica por aumento de volumen en carrillo izquierdo de un año de evolución, sin presencia de síntomas asociados, negando antecedentes de trauma o cirugía en la zona. En el examen clínico se observa lesión nodular subcutánea a nivel de la mucosa yugal izquierda, de un centímetro de diámetro aproximado, bien definida, fluctuante, no hay evidencia de dolor, y presenta color de mucosa normal (Figura 1).

Previa firma de consentimiento informado para el procedimiento, cumpliendo el protocolo de asepsia intraoral y extraoral, se realizó biopsia excisional con abordaje intraoral, incisión en mucosa yugal izquierda, disección roma de la lesión, la cual tenía apariencia encapsulada y contenido blando blanquecino, no se apreció exudado seroso ni hemorrágico, lográndose la extirpación completa tanto de la lesión como de glándulas salivales menores adheridas (Figura 2). Posteriormente se aplicó irrigación profusa con suero fisiológico y se suturó por planos con Vicryl 3,0. La muestra fue embebida de forma inmediata en formol al 10\% y rotulada con fines de estudio histopatológico. Macroscópicamente el espécimen presentó forma irregular, con presencia de anexos cutáneos capilares, color blanquecino, de medidas $1,0 \times 1,0 \times 0,8 \mathrm{~cm}$ (Figura 3).

$\mathrm{Al}$ examen microscópico se observó una cavidad revestida por epitelio escamoso estratificado similar a la epidermis en la cual se observa una capa celular granular bien desarrollada sin atipia, el lumen se encontraba cubierto con ortoqueratina degenerativa (Figura 4).

Se observa también una amplia cavidad revestida por epitelio escamoso estratificado de cinco a siete capas de células, similar a la epidermis, con una capa celular granular bien desarrollada sin atipia, el lumen de esta cavidad lleno de ortoqueratina degenerativa. El epitelio descansaba sobre tejido conectivo fibroso vascularizado, con leve infiltrado inflamatorio lifoplasmocitario. Ausencia de mitosis atípicas, indicando negativo para malignidad (Figura 5). 


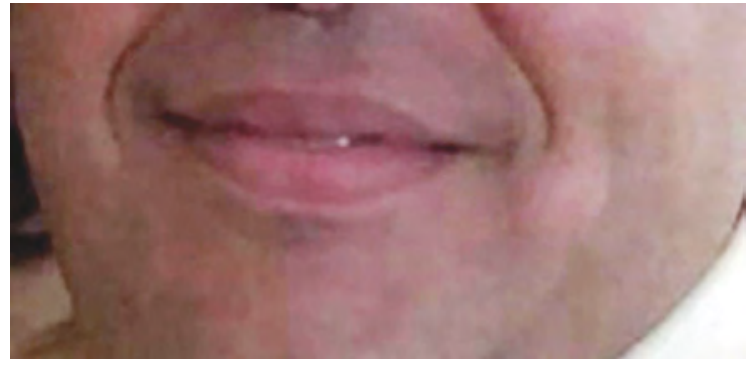

Figura 1. Aspecto clínico del paciente.

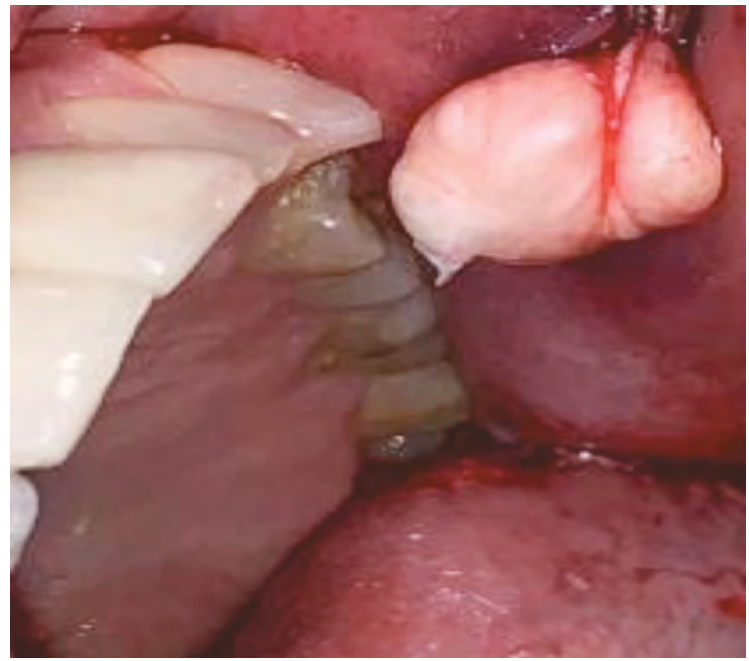

Figura 2. Biopsia excisional. Imagen intraoral durante la realización de la biopsia excisional en la que se observa lesión de color blanquecino, encapsulada por tejido conectivo fibroso, sin drenaje de contenido seroso ni hemorrágico.

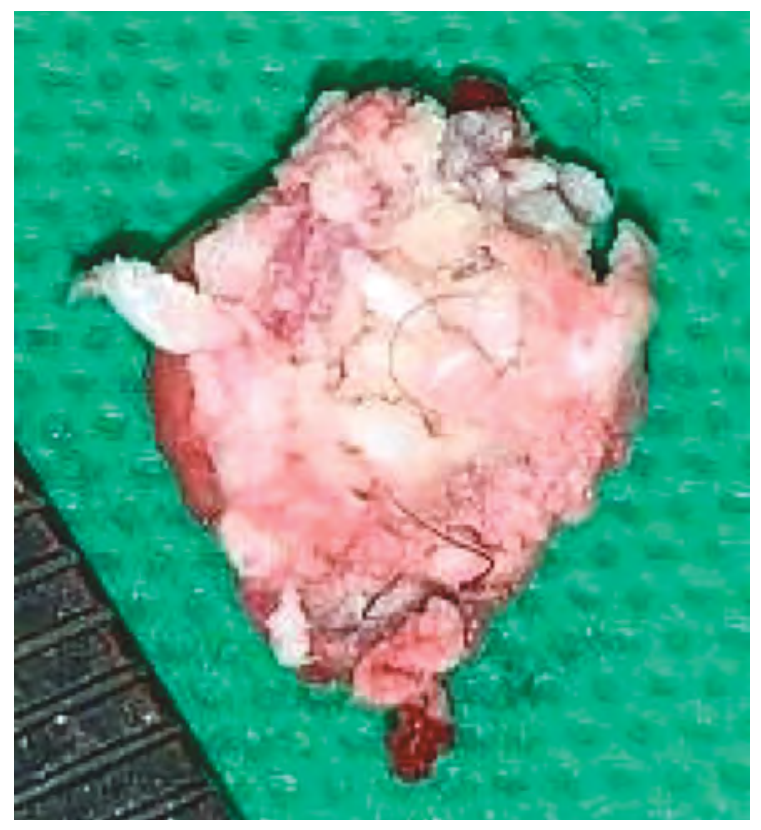

Figura 3. Lesión macroscópica. Imagen del espécimen obtenido posterior a la realización de la biopsia.

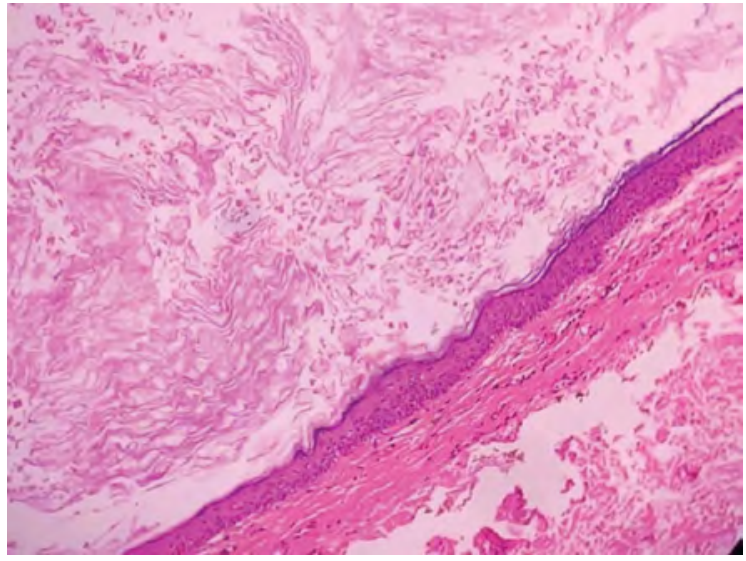

Figura 4. Imagen microscópica en la que se observa capa celular granular y lumen cubierto con ortoqueratina degenerativa. Microfotografía 10X - tinción: hematoxilina / eosina, microscopio Olympus CX23.

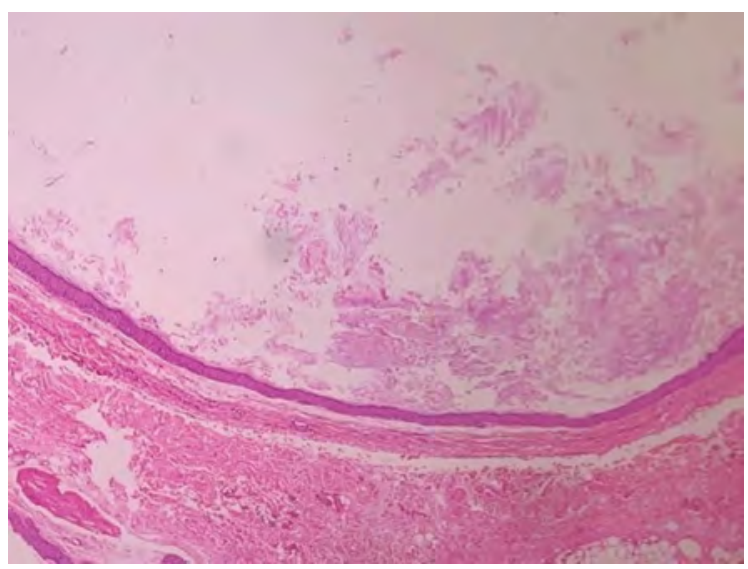

Figura 5. Imagen microscópica en la que se observa epitelio escamoso estratificado que descansa sobre tejido conectivo fibroso vascularizado. Microfotografía 40X - tinción: Hematoxilina / Eosina, microscopio Olympus CX23.

Se informó el resultado del estudio al paciente quien no reportó complicación alguna durante el postoperatorio.

\section{DISCUSIÓN}

Los quistes epidermoides son lesiones poco frecuentes, de naturaleza benigna, y que a nivel de cabeza y cuello generalmente aparecen en el piso de boca ${ }^{2}$. Los hallazgos inflamatorios en cavidad oral podrían generar diversos diagnósticos diferenciales, tales como adenoma pleomórfico, quiste dermoide, mucocele, reacciones granulomatosas de cuerpo extraño a materiales de relleno cosmético ${ }^{5,13-15}$. 
La etiología de los quistes epidermoides es esencialmente desconocida, se considera que pueden ser congénitos, originándose en cualquier punto de desarrollo del cuerpo, donde el tejido ectodérmico podría verse atrapado en la línea de fusión de los procesos embrionarios. Otro factor causal atribuido es de tipo postraumático, donde se comportan como injerto cutáneo, multiplicándose o produciendo una masa de queratina con crecimiento lento por expansión ${ }^{16}$.

En el presente caso al descartar antecedentes de trauma, el diagnóstico presuntivo de quiste dermoide se logró por la presencia de folículos pilosos. Histológicamente, en los quistes epidermoides recientes puede apreciarse numerosas capas de células escamosas y gránulos de queratohialina en el estrato granuloso. Cuando el quiste tiene un tiempo avanzado de evolución, el revestimiento epitelial con frecuencia puede mostrar áreas de atrofia que constituyen uno o dos estratos celulares ${ }^{17-19}$. En el paciente del presente caso, microscópicamente lograron evidenciarse numerosas capas de células revistiendo una amplia cavidad, lo cual pudiese indicar que el quiste era de corta evolución.

En cuanto al tratamiento, lo indicado es la extirpación quirúrgica completa ${ }^{5,8}$, en ocasiones se podría aplicar dexametasona intralesional con el propósito de disminuir el tamaño y facilitar su extracción ${ }^{18}$. En este caso fue un proceso sencillo, debido a que el revestimiento de la lesión facilitó el avance del procedimiento excisional.

Los quistes epidermoides son poco frecuentes en cavidad oral, el tratamiento indicado es la biopsia excisional, generalmente sin presencia de complicaciones quirúrgicas, posquirúrgicas o recurrencias.

\section{Confidencialidad de datos}

El paciente aprobó de forma escrita y voluntaria el uso de fotografías, imágenes diagnósticas y toda la información requerida del procedimiento clínico y la publicación en el presente manuscrito. Esta investigación no violó alguno de los principios establecidos en la Declaración de Helsinki (2000).

Los autores afirman que siguieron los protocolos de su centro de trabajo sobre la publicación de los datos del paciente.

\section{REFERENCIAS}

1. Silveira HA, Almeida LY, Dominguete MHL, Graciano KPP, Bufalino A, León JE. Intraoral epidermoid cyst with extensive elastofibromatous changes: an unusual finding. Oral Maxillofac Surg. 2019;23(4):493-7. DOI: 10.1007/ s10006-019-00785-y.

2. Ueno T, Takayama R, Osada SI, Saeki H. Epidermoid cyst arising on the body of the tongue: case report and literature review. J Nippon Med Sch. 2018;85(6):343-6. DOI: 10.1272/jnms.JNMS.2018_85-56.

3. Wollina U, Langner D, Tchernev G, França K, Lotti T. Epidermoid Cysts - A wide spectrum of clinical presentation and successful treatment by surgery: retrospective 10-year analysis and literature review. J Med Sci. 2018;6(1):28-30. DOI: 10.3889/oamjms.2018.027.

4. Mahalakshmi S, Reddy S, Ramamurthy TK, Shilpa B. Rare Locations of Epidermoid cyst: case reports and review. Ethiop J Health Sci. 2016; 26(6):595-601. DOI:10.4314/ ejhs.v26i6.14.

5. Costa FW, Carvalho FS, Chaves FN, de Almeida S, Alves AP, Patrocinio RM, et al. Epidermoid cyst arising in the buccal mucosa: case report and literature review. Acta Stomatol Croat. 2014;48(4):296-304. DOI: 10.15644/ asc $48 / 4 / 8$.

6. Utumi ER, Araujo JP, Pedron IG, Yonezaki F, Machado GG, Rocha AC. Extensive epidermoid cyst of the submental region. Autopsy Case Rep. 2016;6(2):51-4. DOI: 10.4322/acr.2016.031.

7. Findik Y, Topal O, Senturk MF, Baykul T. Extraoral approach of the surgical treatment of sublingual epidermoid cyst: a case report. J Pak Med Assoc. 2017;67(5):796-8.

8. Puranik SR, Puranik RS, Prakash S, Bimba M. Epidermoid cyst: report of two cases. J Oral Maxillofac Pathol. 2016;20(3):546-60. DOI: 10.4103/0973-029X.190965.

9. Saha A, Das NK, Gharami RC, Chowdhury SN, Datta PK. A clinico- histopathological study of appendageal skin tumors, affecting head and neck region in patients attending the dermatology opd of a tertiary care centre in eastern India. Indian J Dermatol. 2011;56(1):33-6. DOI:10.4103/0019-5154.77548.

10. Costa FW, Carvalho FS, Chaves FN, de Almeida S, Alves AP, Patrocínio RM, et al. Epidermoid cyst arising in the buccal mucosa: case report and literature review. Acta Stomatol Croat. 2015;49(1):65-73. DOI: 10.15644/ asc $49 / 1 / 9$. 
11. Qamar Z, Ikram K, Fatima T. Sublingual epidermoid cyst mimicking as plunging ranula - A case report. Int J Dent Sci Res. 2016;3(1):22-5. DOI:10.1016/j.ijdsr.2015.11.004.

12. De Mendonça JCG, Jardim ECG, Dos Santos CM, Masocatto DC, de Quadros DC, Oliveira MM, et al. Epidermoid cyst: clinical and surgical case report. Ann Maxillofac Surg. 2017;7(1):151-4. DOI: 10.4103/ams.ams_68_16.

13. Seo J, Bruno I, Artico G, Vechio AD, Migliari DA. Oral mucocele of unusual size on the buccal mucosa: clinical presentation and surgical approach. Open Dent J. 2012;6:67-8. DOI: 10.2174/1874210601206010067.

14. Sanchis-Bielsa JM, Bagán JV, Poveda R, Salvador I. Foreign body granulomatous reactions to cosmetic fillers: a clinical study of 15 cases. Oral Surg Oral Med Oral Pathol Oral Radiol Endod. 2009;108(2):237-41. DOI: 10.4317/ jced.50868.
15. Cueva Sánchez MA, Morales Barrera ME, Ramos-Garibay A. Quiste epidérmico de localización poco frecuente. Reporte de un caso. Rev Cent Dermatol Pascua. 2001;10(2):100-2.

16. Santana A, Ferreira O, Clóvis M. Epidermoid cyst: highlights on diagnosis and magnetic resonance imaging features. Rev Odonto Ciênc. 2010;25(2):204-7. DOI: 10.1590/ S1980-65232010000200019.

17. Ettinger RL, Manderson RD. Implantation keratinizing epidermoide cysts. Oral Surg Oral Med Oral Pathol. 1973;36(2):225-30. DOI: 10.1016/00304220(73)90242-9.

18. Ruggeri C, Garcia E, Ianardi F. Quistes epidermoides de cabeza y cuello. Rev Hosp Ital B Aires. 2018;38(1):25-29.

19. Ozan F, Polat HB, Ay S, Goze F. Epidermoid cyst of the buccal mucosa: a case report. J Contemp Dent Pract. 2007;8(3):90-6.

\section{Correos electrónicos de los autores:}

Sandra Milena Buitrago-Rojas: sandra.buitrago@ustabuca.edu.co

Gerson Enrique Cruz-Pérez: gerson.cruz@ustabuca.edu.co 